\title{
Generation of the first Autosomal Dominant Osteopetrosis Type II (ADO2) disease models
}

\author{
Imranul Alam ${ }^{1}$, Amie K. Gray², Kang Chu², Shoji Ichikawa², Khalid S. Mohammad ${ }^{2}$, Marta \\ Capannolo ${ }^{4}$, Mattia Capulli ${ }^{4}$, Antonio Maurizi ${ }^{4}$, Maurizio Muraca ${ }^{5}$, Anna Teti ${ }^{4}$, Michael J. \\ Econs $^{2,3}$, and Andrea Del Fattore ${ }^{5}$ \\ ${ }^{1}$ Department of Orthopedic Surgery, Indiana University, 541 North Clinical Drive, Indianapolis, IN \\ 46202, USA \\ 2Department of Medicine, Indiana University, 541 North Clinical Drive, Indianapolis, IN 46202 , \\ USA \\ ${ }^{3}$ Department of Medical and Molecular Genetics, Indiana University, 541 North Clinical Drive, \\ Indianapolis, IN 46202, USA \\ ${ }^{4}$ Department of Biotechnological and Applied Clinical Sciences, University of L'Aquila, Via Vetoio \\ - Coppito 2, 67100 L'Aquila, Italy. \\ ${ }^{5}$ Regenerative Medicine Unit, Bambino Gesù Children's Hospital, Istituto di Ricovero e Cura a \\ Carattere Scientifico, piazza Sant'Onofrio 4, 00165 Rome, Italy.
}

\section{Abstract}

Autosomal Dominant Osteopetrosis Type II (ADO2) is a heritable osteosclerotic disorder dependent on osteoclast impairment. In most patients it results from heterozygous missense mutations in the chloride channel $7(C L C N 7)$ gene, encoding for a $2 \mathrm{Cl}^{-} / 1 \mathrm{H}^{+}$antiporter. By a knock-in strategy inserting a missense mutation in the $\mathrm{Clcn} 7$ gene, our two research groups independently generated mouse models of $\mathrm{ADO} 2$ on different genetic backgrounds carrying the homolog of the most frequent heterozygous mutation (p.G213R) in the $\mathrm{Clcn} 7$ gene found in humans. Our results demonstrate that the heterozygous model holds true presenting with higher bone mass, increased numbers of poorly resorbing osteoclasts and a lethal phenotype in the homozygous state. Considerable variability is observed in the heterozygous mice according with the mouse background, suggesting that modifier genes could influence the penetrance of the disease gene.

\section{Keywords}

Osteopetrosis; Autosomal dominant osteopetrosis; Osteoclast; Chloride channel 7; Mouse model

\footnotetext{
(C) 2013 Elsevier Inc. All rights reserved.

Corresponding Author: Anna Teti, PhD Department of Biotechnological and Applied Clinical Sciences University of L'Aquila Via Vetoio - Coppito 267100 L’Aquila, Italy Tel +390862433511/13 Fax +390862433523 teti@univaq.it/mecons@iupui.edu.

Publisher's Disclaimer: This is a PDF file of an unedited manuscript that has been accepted for publication. As a service to our customers we are providing this early version of the manuscript. The manuscript will undergo copyediting, typesetting, and review of the resulting proof before it is published in its final citable form. Please note that during the production process errors may be discovered which could affect the content, and all legal disclaimers that apply to the journal pertain.

Conflict of interest statement: All authors state that they have no conflicts of interest.
} 


\section{Introduction}

Autosomal Dominant Osteopetrosis type 2 (ADO2) is a genetic bone disease affecting 5.5 in 100,000 people [1] and is characterized by a wide range of symptoms and severity [2-3]. Although considered a mild type of osteopetrosis, rarely this disease can be lethal [2]. In the most severe forms, patients present with extensive osteosclerosis, especially of the skull base, pelvis and vertebrae, and experience multiple fractures, osteomyelitis, hematologic failure and sensorial symptoms, including impaired vision and hearing [4]. The disease is presently incurable and, in contrast to the autosomal recessive forms [5], there is no murine model for the dominant type to meet the biomedical need to understand the pathogenic molecular mechanisms and test treatments.

So far only mutations in one gene, $C L C N 7$, are known to be involved in ADO2 affecting the majority of patients [6], with approximately $66 \%$ penetrance [7]. The CLCN7 gene codes for the $\mathrm{ClC}-7$ subunit, which assembles into a homodimer. The $\mathrm{ClC}-7$ subunit is characterized by 11 transmembrane domains and cytosolic $\mathrm{N}$-terminal and $\mathrm{C}$-terminal tails [8]. Initially classified as a chloride channel, the protein is now confirmed to serve as a $\mathrm{Cl}^{-} / \mathrm{H}^{+}$antiporter with a 2:1 stoichiometry [9]. ClC-7 resides in lysosomes in association with an accessory $\beta$ subunit called Ostm1, required for its function [10]. The ClC-7/Ostm1 complex is coinserted with the vesicular $\mathrm{H}(+)$-ATPase into the acid-secreting ruffled border membrane of osteoclasts and is essential to charge balance the outward proton transport that enables the dissolution of the mineralized matrix by acidifying the resorption lacuna [11].

Bi-allelic loss-of-function mutations of the CLCN7 gene and knock-out of its murine homolog induce a severe autosomal recessive osteopetrosis [12]. Heterozygous dominant missense mutations are instead responsible of human ADO2 [6]. Among the many mutations identified so far to work in a dominant fashion, the p.G215R substitution is the most frequent and better characterized [3,6]. It has been proposed that this amino acid change does not abolish the ClC-7 exchange activity but rather severely impairs ClC-7 cellular distribution, with the protein essentially retained in the endoplasmic reticulum [13]. This result, however, contrasts the previous finding that p.G215R ClC-7 is normally distributed in ADO2 osteoclasts [14], suggesting that more work and better models are necessary to uncover all $\mathrm{ClC}-7$ functions in a pathophysiologic context.

Indeed, no mouse $\mathrm{ADO} 2$ models existed so far for the single allele mutation of $\mathrm{Clcn} 7$ gene that could address the pathogenesis of the disease, the mechanisms of incomplete penetrance and the understanding of the $\mathrm{ClC}-7$ function. Our two groups, independently, filled this gap by creating a heterozygous mouse model of the p.G215R mutation on the C57BL6/J (B6) background. We also generated models of phenotypic variability cross-breeding B6 ADO2 mice with mice of 129Sv (129), DBA/2J (D2), BALB/cJ (Balb/c) and Crl:CD-1 (CD1) genetic backgrounds. We observed that these models hold true and recapitulate features of the human $\mathrm{ADO} 2$, thus representing new tools to deeply investigate the underlying cellular and molecular mechanisms and test treatments.

\section{Materials and methods}

\subsection{Animals}

Mice were housed in polycarbonate cages in a vivarium maintained on a 12-h light and 12-h dark cycle and were fed a regular diet and water ad libitum. Procedures involving animals and their care have been conducted in conformity with national and international laws and policies (EEC Council Directive 86/609; Italian Legislative Decree 116/92; NIH guide for the Care and Use of Laboratory Animals), with Institutional approval at the University of 
L'Aquila and according to the guidelines of the Indiana University Animal Care and Use Committee (IACUC).

\subsection{Generation of the p.G213R-Clcn7 knock-in mouse (University of L'Aquila)}

The gene targeting construct was obtained by cloning the $C l c n 7$ exon 7 and the $5^{\prime}$ and $3^{\prime}$ homologous regions in the pFlrt1 vector (Figure 1). The p.G213R mutation was created inserting a G-A transition at DNA position 14365 using the QuikChange II XL Site-Directed Mutagenesis Kit (Stratagene, La Jolla, CA) and primers containing the desired mutation. The gene targeting construct was linearised by NotI digestion and $35 \mu \mathrm{g}$ of the linearised plasmid were electroporated into 15 million mouse Embryonic Stem (ES) cells derived from a 129S2 mouse. The electroporated cells were subjected to positive and negative selection by neomycin and gancyclovir, respectively. The $\sim 280$ clones obtained were first subjected to Southern blot screening to identify those correctly recombined at $3^{\prime}$. Then, $5^{\prime}$ screening was performed by PCR, using primers annealing to pFlrt1 vector sequences just upstream and downstream the region containing exon 7 (Figure 2). Three positive clones (1C7, 1E9, 2C6) were injected into mouse blastocysts and these were implanted into B6 foster mothers to generate chimeras. Chimeras were bred with wild-type B6 mice to obtain F1 heterozygous knock-in mice, further bred with FlpE transgenic mice to remove the exogenous Neo cassette from the genomic DNA. After Neo cassette removal, knock-in mice were bred with B6 and CD1 wild-type mice. Mice were sacrificed by cervical dislocation, and bones, brains and sera collected for analyses. Results obtained with B6 mice derived from the 1E9 ES clone are shown.

\subsection{Generation of the p.G213R-Clcn7 knock-in mouse (Indiana University)}

We used homologous recombination technique to insert the p.G213R mutation in exon 7 and CreloxP recombination strategy to remove the positive selection gene (neomycin) from the targeted allele (Figure 3). The wild type $\mathrm{Clcn} 7$ targeting fragment was inserted into pcDNA3.1 cloning plasmid and site directed mutagenesis was performed to create the p.G213R mutation in the exon 7 using the QuikChange ${ }^{\circledR}$ kit from Stratagene (La Jolla, CA). To avoid potential lethality in heterozygous offspring, we also introduced a nonsense mutation in exon 6 in the target construct to stop $\mathrm{Clcn} 7$ transcription. The final linearized target construct, contained exon 6 with a stop codon flanked by loxP sites, the neomycin resistance gene also flanked by loxP sites, as well as the p.G213R mutation in exon 7. To decrease random targeting of our construct in ES cells, we subcloned the phosphoglycerate kinase promoter driven-diphtheria toxin A fragment gene (PGKDta) into the $3^{\prime}$ end of the Clcn 7 targeting construct. This construct $(50 \mu \mathrm{g})$ was inserted into $5 \times 10^{7}$ embryonic stem cells (CCE916) from 129Sv mouse via electroporation for homologous recombination. Approximately, two hundred colonies resistant to G418 were screened by PCR using primers at $5^{\prime}$-end (P1-F and P1-R) and at $3^{\prime}$-end (P2-F and P2-R). Positive clones (only mutants with bands 3626 and 5969 bp at $5^{\prime}$-end and $3^{\prime}$-end, respectively) were injected into B6 blastocysts foster mothers to generate chimeras, which were bred with wild-type B6 mice to obtain F1 heterozygous knock-in mice. These mice were further bred with EIIa-Cre transgenic mice to generate progeny with removal of exon 6 carrying the null mutation and PKG-neo cassette from the genomic DNA, which were confirmed by using primers spanning all loxP sites (P3-F and P3-R). Mutation in exon 7 was determined using restriction fragment length polymorphism analysis after PCR. Finally, B6 knock-in mice were bred with 129, D2 and Balb/c wild-type mice.

\subsection{Dual energy X-ray absorptiometry (DXA) (Indiana University)}

The whole body aBMD $\left(\mathrm{g} / \mathrm{cm}^{2}\right)$ was measured using DXA (PIXImus II mouse densitometer; Lunar Corp., Madison, WI, USA) with ultra-high resolution $(0.18 \times 0.18 \mathrm{~mm} / \mathrm{pixel})$. The 
machine was calibrated each time before DXA scanning using a phantom supplied by the manufacturer. The scans were performed with the mice in a prone position, with each limb spread on a plastic tray. The global window was defined as the whole-body image minus the calvarium, mandible, and teeth.

\subsection{X-ray analysis (University of L'Aquila)}

$\mathrm{X}$-ray analysis (36 KPV for $10 \mathrm{sec}$ ) was performed using a Cabinet X-ray system (Faxitron X-Ray Corp., Buffalo Grove, IL). Radiographs were scanned using the Bio-Rad scanning densitometer (Hercules, CA).

\subsection{Micro Computer Tomography $(\mu \mathrm{CT})$ analysis (University of L'Aquila)}

Femurs and vertebrae of B6 and CD1 mice were fixed in buffered formalin, mounted in a plastic tube wrapped in moistened paper tissue to prevent drying during scanning. The bones were imaged in the SkyScan 1174 with a voxel size of $6 \mu \mathrm{m}$. The applied x-ray voltage was $50 \mathrm{kV}$. The scans were over 180 degrees with a 0.3 degree rotation step. Reconstruction was carried out employing a modified Feldkamp algorithm using the Skyscan Nrecon software. Beam hardening correction and Fourier transform-based ring artefact reduction were applied to the reconstruction. 3D and 2D morphometric parameters were calculated for the trabecular bone of selected regions of interest, 100 slices $(0.6 \mathrm{~mm})$ from the growth plate extending for 350 slices $(2.1 \mathrm{~mm})$ below. Threshold values were applied for segmenting trabecular bone corresponding to BMD values of $0.6 / \mathrm{cm}^{3} \mathrm{CaHA}$. 3D parameters were based on analysis of a Marching Cubes type model with a rendered surface. Calculation of all 2D areas and perimeters was based on the Pratt algorithm. Morphometric parameters were measured by CT-analyzer. Structure thickness in 3D was calculated using the local thickness or "sphere-fitting" method. Model construction was performed by the "Double time cubes" method, a modification of the Marching cubes method. Nomenclature, symbols and units of structural bone variables are those suggested by Bouxsein at al. [15].

\section{7 $\mu \mathrm{CT}$ analysis (Indiana University)}

The femurs of 129, D2 and Balb/c were dissected out from each animal and transferred to $70 \%$ ethyl alcohol and stored at $4^{\circ} \mathrm{C}$ until $\mu \mathrm{CT}$ analyses. Each femur was scanned with a high resolution $\mu$ CT scanner (vivaCT 40, Scanco Medical AG, Brüttisellen, Switzerland) with an isotropic voxel size of $10.5 \mu \mathrm{m}^{3}$. Before scanning, the CT-scanner was calibrated using a phantom according to the manufacturer's recommendation. From the scout-view the growth plate location was identified and trabecular bone measurements consisting of 200 slices $(2.1 \mathrm{~mm})$ was done from about $1 \mathrm{~mm}$ below the growth plate. The settings for each scanning included peak X-ray potential of $55 \mathrm{kVp}$, X-ray intensity of $72 \mu \mathrm{A}, 1000$ projections per 180 degrees, image resolution of $2048 \times 2048$ pixels, and integration time of 200 milliseconds. After scanning, contouring was achieved manually a few pixels away from the endocortical surface to delineating the trabecular bone. Image processing of all scans included Gaussian filtering and segmentation by using global thresholds, above which all pixels are considered bone, and below which all pixels are considered non-mineralized tissues ( $\operatorname{sigma}=1$, support $=2$, threshold density $=170$ ). The same filtering and segmentation values were used for each bone for all trabecular bone measurements. Finally, 3D and 2D morphometric evaluations were performed for the trabecular bone from each scan, and bone volume and structural parameters were determined [15].

\subsection{Histology and histomorphometry (University of L'Aquila)}

Brain and cerebellum were fixed in $4 \%$ formaldehyde, in $0.1 \mathrm{M}$ phosphate buffer, $\mathrm{pH} 7.2$, dehydrated in ethanol and processed for paraffin embedding. Tibiae were fixed in buffered $4 \%$ formaldehyde, processed for paraffin embedding with previous decalcification, or for 
glycol-methacrylate embedding without decalcification, respectively. Histomorphometric measurements were carried out on 2 to $5 \mu \mathrm{m}$ thick sections, with an interactive image analysis system (IAS 2000, Delta Sistemi, Rome, Italy). Nomenclature, symbols and units of morphometric bone variables are those suggested by Dempster et al. [16].

\subsection{Serum biomarkers (University of L'Aquila and Indiana University)}

Mouse serum levels of collagen type 1 cross-linked C-telopeptide (CTX), Tartrate-Resistant Acid Phosphatase (TRAcP) 5b isoform, alkaline phosphatase and osteocalcin were measured by Enzyme-Linked ImmunoSorbent (ELISA) kits according to the manufacturers' instructions (Immunodiagnostics Systems, AZ, USA, Randox Rx Daytona Analyzer, WV, USA and Biomedical Technologies Inc., MA, USA). Serum calcium, phosphorus, creatine kinase muscle-brain and biomarkers of diseases were measured using the Reflotron kits (Roche Diagnostics, Manheim, Germany), and the Randox Rx kit (Daytona Analyzer, WV, USA).

\subsection{Serum glucose concentration and glucose tolerance test}

Mice maintained on normal diet, were subjected to the glucose tolerance test following $16 \mathrm{~h}$ overnight fasting. Briefly, fasted glucose was measured at time 0 using the Accu-Chek Aviva System (Roche Diagnostics, Manheim, Germany). Then $2 \mathrm{gr} / \mathrm{Kg}$ dose of glucose was administrated by i.p. injection. Blood glucose levels were then measured as above after 30 , 60, 90 and 120 minutes from injection.

\subsection{Evaluation of TRACP positive mononuclear cells in freshly isolated bone marrow and osteoclast preparations (University of L'Aquila)}

Blood mononuclear cells were prepared from bone marrow stratified over a solution of Histopaque ${ }^{\circledR}-1077$ (Sigma, MO, USA) and centrifuged at $400 g$ for 30 minutes. 'Buffy coat' cells were collected and re-suspended in Dulbecco's modified Minimum Essential Medium (DMEM) containing $4 \mathrm{mM} \mathrm{L}$-glutamine, $100 \mathrm{U} / \mathrm{ml}$ penicillin, $100 \mathrm{mg} / \mathrm{ml}$ streptomycin, and $10 \%$ Fetal Bovine Serum (FBS). Then $10^{6}$ cells $/ \mathrm{cm}^{2}$ were plated in cell culture dishes and incubated at $37^{\circ} \mathrm{C}$ in a humidified atmosphere with $5 \% \mathrm{CO}_{2}$. After 3 hours, cell cultures were rinsed to remove non-adherent cells. Some cultures were fixed immediately to evaluate the osteoclast precursors present in the bone marrow. Others were maintained in the same medium in the presence of $50 \mathrm{ng} / \mathrm{ml}$ recombinant human Macrophage-Colony Stimulating Factor (M-CSF) and $120 \mathrm{ng} / \mathrm{ml}$ recombinant human Receptor Activator of NF- $\kappa B$ Ligand (RANKL) for 7 days. Osteoclasts and osteoclast precursors were detected by cytochemical staining for TRAcP [Acid Phosphatase, Leukocyte (TRAP) Kit, Sigma, MO, USA].

\subsection{Bone resorption assay (University of L'Aquila)}

Bone marrow mononuclear cells were cultured in 96 multiwell plates containing bovine bone slices (Pantec, Torino, Italy) and differentiated into osteoclasts as described above. Bone resorption was determined by the pit assay [17].

\subsection{Statistical analysis}

Quantitative data were expressed as mean \pm s.d. Statistical differences were identified by the unpaired Student's $t$ test using the statistical software package StatView (Abacus Concepts, Inc., Berkeley, CA). The level of significance was set at p-value $<0.05$.

\section{Results}

We generated the p.G213R Clcn7 ADO2 mouse model on the B6 background (Figure 1). B6 ADO2 mice were then cross-bred with the 129, D2, Balb/c and CD1 strains to produce 
additional ADO2 mouse models. B6 and CD1 p.G213R Clcn7 homozygous mice were also obtained and analysed.

\subsection{Phenotype of p.G213R Clcn7 homozygous mice}

Homozygous B6 (Figure 2) and CD1 (Supplementary Figure 1) mice were smaller (Figure 2A,B; Supplementary Figure 1A), lacked tooth eruption (Figure 2C; Supplementary Figure 1B) and died within 30 days of age (Figure 2D). Feeding them with soft chow did not improve survival. Homozygous B6 mice showed a severe osteopetrotic phenotype (Figure 2E-I), with fibrotic bone marrow (Figure 2E) and extensive neurodegeneration in the hippocampal regions and cerebellar cortex (Figure 3A,B), recapitulating the mouse $\mathrm{Clcn} 7$ gene knock-out model as well as the human autosomal recessive disease observed in a large part of patients $[12,15]$. Similar severe osteopetrotic phenotype was observed in homozygous CD1 mice (Supplementary Figure 1C,D).

\subsection{Phenotype of p.G213R Clcn7 heterozygous B6 mice}

Heterozygous B6 ADO2 mice were born at the expected Mendelian frequency. They were vital and fertile and showed unremarkable size, body weight and tooth eruption (Figure 2AC). They showed radiological, histological and bone morphometric signs of a milder form of osteopetrosis (Figure 2E-I), while no neurodegeneration was apparent in the hippocampal regions and cerebellar cortex either in young (age 1 month, Figure 3A,B) or in elderly mice (age 12 months, Figure 3C,D).

\subsection{Bone phenotype of adult p.G213R Clcn7 heterozygous B6 mice}

Adult (3 months old) heterozygous B6 mice had a significantly higher whole body areal Bone Mineral Density (aBMD) than wild-type mice $(+4 \%, \mathrm{p}<0.05)$. They presented with greater distal femur trabecular bone volume, trabecular number and separation, and no changes in trabecular thickness (Figure 4A-E). A similar phenotype was observed even in tibiae (Figure 4F) and vertebrae (Figure 4G-K). Increased bone mass was persistent with time as shown by analysis of bone structural parameters in aging mice (Table 1). Results were similar in male and female mice, although females had a lower bone mass and more pronounced BV/TV elevation than males (Table 2).

\subsection{Cellular and dynamic parameters}

Histochemical examination of 3 months old heterozygous mice demonstrated a marked increase of calvarial bone whole mount staining for the osteoclast marker TRAcP (Figure $5 \mathrm{~A}$ ), and higher osteoclast number (Figure 5B) and osteoclast surface/bone surface (Figure 5C) in the proximal tibia compared to wild-type mice. Nevertheless, a reduction of bone resorption was predicted by a trend of decrease of the serum level of the bone resorption marker CTX, normalized for the serum TRAcP (Figure 5D). In contrast, serum level of a bone formation marker, osteocalcin, as well as osteoblast, bone formation, osteoid and growth plate histomorphometric parameters were similar to wild-type (Figure 5E-J), suggesting no involvement of osteoblasts or chondrocytes, nor an osteopetrorickets phenotype.

\subsection{In vitro osteoclast cultures}

The bone marrow from $\mathrm{B} 6 \mathrm{ADO} 2$ mice showed increased TRAcP-positive mononuclear osteoclast precursors (Figure 6A) versus wild-type mice. When cultured with M-CSF and RANKL these precursors formed a higher number of osteoclasts with normal morphology (Figure 6B,C) and a trend to increase of size (number of nuclei/osteoclast, WT: $25.2 \pm 3.49$, $\mathrm{n}=5$; HT: $30.29 \pm 4.42, \mathrm{n}=7, \mathrm{p}=0.06$ ). Importantly, similar to patients [2,22], these osteoclasts 
showed reduction of resorption pit formation (Figure 6D), confirming a cell autonomous impairment of bone breakdown.

\subsection{Laboratory tests}

Laboratory tests showed that 3 months old B6 ADO2 mice had normal hematologic parameters (Supplementary Table 1) and serum biomarkers of pancreas (amylase), liver (glutamic-oxaloacetic transaminase, alanine transaminase, alkaline phosphatase) and kidney (potassium) diseases (Supplementary Table 2). They also showed normal calcium, phosphorous, creatine kinase-muscle brain (Supplementary Table 2) and glucose concentration and responded normally to the glucose tolerance test (Supplementary Figure 2). In contrast, serum level of $\mathrm{PTH}$ was higher in $\mathrm{B} 6 \mathrm{ADO} 2$ compared to wild-type mice [PTH $(1-84)(\mathrm{pg} / \mathrm{ml})$ : wild-type mice: $66.55 \pm 53.72$; B6 ADO2 mice: $131.85 \pm 75.58$; mean $\pm \mathrm{SD} ; \mathrm{n}=6-11 ; \mathrm{p}=0.045]$.

\subsection{Phenotype of p.G213R Clcn7 heterozygous 129, D2, Balb/c and CD1 mice}

The penetrance of ADO2 in human is approximately $66 \%$ and severity varies considerably $[3,6]$. To create models of phenotypic variability we cross-bred B6 ADO2 mice with mice of 129, D2, Balb/c and CD1 genetic backgrounds. As with B6 ADO2 mice, heterozygous mice of 129, D2, Balb/c and CD1 strains were also born at the expected Mendelian frequency. These mice were fertile and had similar body size as wild-type mice, normal tooth eruption and radiological, histological and bone morphometric signs of a milder form of osteopetrosis.

We focused on bone phenotypes, hematologic tests and serum level of bone resorption marker in the heterozygous ADO2 mice. Compared to wild-type, the whole aBMD at 3 months of age were very high in ADO2 mice on 129 background (Table 3). ADO2 mice on D2 background also had significantly higher whole aBMD (Table 3). Trabecular bone volume and trabecular number were significantly higher and trabecular separation was significantly lower at distal femur in ADO2 mice on 129 and D2 backgrounds, while trabecular bone volume was higher in $\mathrm{ADO} 2$ mice on $\mathrm{Balb} / \mathrm{c}$ background and changes were not significant in $\mathrm{ADO} 2$ mice on CD1 background (Table 4). CTX/TRAcP ratio was significantly lower in all cross-bred ADO2 backgrounds, except the D2 (Table 5). Serum ALP was similar in ADO2 mice on 129 and Balb/c background but D2 mice showed higher $(\mathrm{p}<0.05)$ ALP compared to wild-type mice (Supplementary Figure 3). In addition, serum osteocalcin level was not significantly different in ADO2 mice on 129, Balb/c and D2 background (Supplementary Figure 3). Finally, we found similar serum calcium and phosphorous level (Supplementary Figure 4), red blood count, hemoglobin and hematocrit (Supplementary Table 3) in ADO2 mice on different background compared to the wild-type mice.

\section{Discussion}

Our results demonstrate that we have generated the first bona fide mouse models of ADO2 caused by the heterozygous p.G213R Clcn 7 gene mutation. This genotype induces a genuine osteopetrotic phenotype as shown by a severe course in homozygous mice and a milder course, similar to ADO2, in heterozygous mice that phenocopy the human disease.

ADO2, or Albers-Schönberg disease, represents the most frequent form of osteopetrosis and, although this disorder increases bone mineral density, the bone is of poor quality and patients frequently present with fractures, bone pain and chronic osteomyelitis $[1-4,18,19]$. Some patients are wheelchair bound due to multiple fractures at pelvis, hip or femur. Sometimes severe anaemia with extramedullary haematopoiesis can be a particularly 
troublesome complication of the disease. Therefore, this disease may cause substantial suffering and impairs the quality of life of patients and their families.

ADO2 is generally diagnosed in adolescence or early adulthood [20], most often due to pathological fractures that require radiologic evaluation which unveils unusually dense but fragile bones. Rarely the disease is diagnosed in infancy or childhood and these forms have been confused with autosomal recessive osteopetrosis until a genetic test became available [20]. While bone marrow transplantation is used for many forms of autosomal recessive osteopetrosis, it is not generally performed for ADO2. Therapy for ADO2 patients is generally palliative consisting of pain control, surgical interventions to repair fractures, treat osteomyelitis or dental problems, and decompress nerves, which is at least in part, due to the lack of an appropriate animal model of ADO2.

Currently, several mouse models of osteopetrosis are available, generated by either spontaneous mutations or genetic manipulation of the involved genes [5,19]. While some of these models are reminiscent of human forms of osteopetrosis [5,19], all have autosomal recessive inheritance. Loss-of-function mutations of the CLCN7 gene and knock-out of its murine homolog induce a severe autosomal recessive osteopetrosis [11]. In the Clcn7-/mice a serious lysosomal storage disease is also observed leading to neuron loss in the forebrain, in the cerebellum and in the retina [21]. This phenotype is shared by the $g l / g l$ mice, carrying a mutation of the homolog of the human OSTM1 gene [22] and is observed also in patients harbouring recessive mutations of the CLCN7 gene although, in contrast with the OSTM1 mutants, not all of them are neuropathic [23]. A recent report has shown that lysosomal pathology and osteopetrosis in ClC-7 mutants are associated to loss of proton-driven accumulation of chloride in lysosomes. In fact, a point mutation (p.G245A) inserted in the mouse $\mathrm{Clcn} 7$ gene, that converted ClC-7 into an uncoupled chloride conductor (clcn7unc/unc mice) induced a lysosomal storage disease similar to that of clcn7-/- mice [24]. Since the osteopetrosis in the $c l c n 7 u n c / u n c$ mice was milder, it was concluded that some roles of $2 \mathrm{Cl}^{-} / 1 \mathrm{H}^{+}$exchange are taken over by the $\mathrm{Cl}^{-}$conductance. Unfortunately, there is no mouse model for the dominant type of osteopetrosis. Thus, a true animal model of $\mathrm{ADO} 2$ would be extremely valuable not only to understand better the pathophysiology of this disease but also to identify suitable therapeutic interventions for $\mathrm{ADO} 2$ patients. Since almost all ADO2 patients harbour missense mutations in the CLCN7 gene and the p.G215R amino acid substitution is among the most common mutations, our two groups independently adopted a knock-in strategy to introduce the homolog of the human mutation (p.G213R) in mice on different genetic backgrounds.

While homozygous B6 mice showed a severe osteopetrotic phenotype and died within a month, even if fed with a soft chow diet, the heterozygous mice presented signs of a milder form of this disease. The osteopetrotic phenotype was observed both in growing (1 month), adult ( 3 months) and aging (6 and 12 months) B6 ADO2 mice, with a more evident phenotype in females than in males. In addition, heterozygous ADO2 mice showed higher osteoclast number, size and surface but lower osteoclast activity. These results suggest that, as in patients [18], the varying degree of bone phenotypes observed in ADO2 mice is due to osteoclast specific properties (defective osteoclast resorption). The mechanism inducing increased osteoclast numbers is matter for speculation, but it could involve increased PTH levels as observed in our $\mathrm{B} 6 \mathrm{ADO} 2$ mice and in $\mathrm{ADO} 2$ patients (26).

Since no changes were detected in our mice for osteoblast, bone formation and osteoid parameters, it is tempting to exclude osteoblast involvement or mineralization defects in ADO2. However, in the setting of markedly reduced resorption, "normal" bone formation is inappropriate. Thus, although ADO2 results primarily from defective osteoclastic bone 
resorption, there may be a subtle relative "increase" in formation compared to what is physiologically appropriate.

Intriguing aspects of $C L C N 7$-dependent $\mathrm{ADO} 2$ are the incomplete penetrance (66\%) of the mutations [7] and the marked phenotypic variability among patients with the same mutation $[3,27]$. Affected subjects can either be asymptomatic carriers or have symptoms that range from very mild to severe $[3,7,23,27]$. The molecular mechanisms of this heterogeneity are still unknown, however, it is likely that genetic factors other than $C L C N 7$ gene modify disease severity and penetrance. Our data in mice, demonstrating a wide range of phenotypic variability in $\mathrm{ADO} 2$ mice on different genetic backgrounds, confirm and extend those observed in patients. For example, mutant and wild-type mice from the 129, D2 and Balb/c strains were kept in identical environmental conditions, yet BV/TV in mutant mice on the 129 strain was almost double that of wild-type while it was only $44 \%$ and $20 \%$ increased on the D2 and Balb/c background, respectively. These data support the concept that genetic elements outside of the $C L C N 7$ gene itself play a critical role in determining the phenotype. Investigating these determinants could be critical to identify targets for therapy that may allow the transition of a severe form to a milder form. However, whether our findings result from the presence of modifier genes or other genetic elements (i.e. noncoding RNA) or a combination of different factors requires further investigation.

In this study, we adopted a knock-in approach to generate ADO2 mouse models rather than producing a transgenic mouse model overexpressing the mutant gene, because the knock-in approach will allow us to more extensively explore the pathophysiology of this disease. Although technically less complex, a transgenic mouse would not allow identification of genetic modifiers that influence phenotypic variability as differences in expression of the mutant gene will vary between different lines of mice, which will have a tremendous effect on disease severity. Furthermore, in order to facilitate the identification of modifier genes or other genetic factors we generated $\mathrm{ADO} 2$ mice on those genetic backgrounds that show substantial differences of bone density measurements. Importantly, sequence data from some of these strains are already available (B6 and D2) or will be available soon.

There are some limitations in this study. One of the limitations is that all ADO2 mouse models except B6 are not bred yet at complete homozygosity (N10). While 129 and D2 $\mathrm{ADO} 2$ mice are at $5^{\text {th }}$ generation (N5) which reflects about $95 \%$ homozygosity, Balb/c and $\mathrm{CD} 1$ mice are still at F1 generation. Thus, the phenotypic difference we observed in F1 mice might be significantly different when breeding these mice reach to complete homozygosity. Furthermore, except B6 ADO2 mice, we obtained our data only at 3 months of age. Whether age has any effect for the severity of the disease deserves further investigation.

In conclusion, we have generated reasonable mouse models of $\mathrm{Clcn} 7$-dependent $\mathrm{ADO} 2$ that echo the human disease and its high phenotypic variability. These models will help to understand the pathogenesis of this form of osteopetrosis, identify the genes that influence penetrance and severity, and test innovative therapies to treat this presently incurable disease.

\section{Supplementary Material}

Refer to Web version on PubMed Central for supplementary material.

\section{Acknowledgments}

We are indebted to the "Core Facility for Conditional Mutagenesis", San Raffaele Institute, Milan, Italy, for their excellent assistance in p.G213R-Clcn7 knock-in mouse generation. We also thank Dr. Alessandra Di Muzio for her valuable technical assistance and Dr. Rita Di Massimo for editing the manuscript. This work was supported by the 
Telethon grant \#GGP09018 to AT, by an European Calcified Tissue Society PhD studentship to MC, by a grant from the "Società Italiana dell'Osteoporosi, del Metabolismo Minerale e delle Malattie dello Scheletro" to ADF, and National Institute of Health (NIH) grants R21 AR054744 and R01 AG041517 to MJE.

\section{References}

[1]. Bollerslev J. Osteopetrosis, A genetic and epidemiological study. Clin. Genet. 1987; 31:86-90. [PubMed: 3829443]

[2]. Del Fattore A, Peruzzi B, Rucci N, Recchia I, Cappariello A, Longo M, Fortunati D, Ballanti P, Iacobini M, Luciani M, Devito R, Pinto R, Caniglia M, Lanino E, Messina C, Cesaro S, Letizia C, Bianchini G, Fryssira H, Grabowski P, Shaw N, Bishop N, Hughes D, Kapur RP, Datta HK, Taranta A, Fornari R, Migliaccio S, Teti A. Clinical, genetic, and cellular analysis of 49 osteopetrotic patients: implications for diagnosis and treatment. J. Med. Genet. 2006; 43:315325. [PubMed: 16118345]

[3]. Waguespack SG, Hui SL, Dimeglio LA, Econs MJ. Autosomal dominant osteopetrosis: clinical severity and natural history of 94 subjects with a chloride channel 7 gene mutation. J. Clin. Endocrinol. Metab. 2007; 92:771-778. [PubMed: 17164308]

[4]. Bollerslev J, Mosekilde L. Autosomal dominant osteopetrosis. Clin. Orthop. Relat. Res. 1993; 294:45-51. [PubMed: 8358946]

[5]. Van Wesenbeeck L, Van Hul W. Lessons from osteopetrotic mutations in animals: impact on our current understanding of osteoclast biology. Crit. Rev. Eukaryot. Gene Expr. 2005; 15:133-162. [PubMed: 16022633]

[6]. Cleiren E, Bénichou O, Van Hul E, Gram J, Bollerslev J, Singer FR, Beaverson K, Aledo A, Whyte MP, Yoneyama T, deVernejoul MC, Van Hul W. Albers-Schönberg disease (autosomal dominant osteopetrosis, type II) results from mutations in the $\mathrm{ClCN} 7$ chloride channel gene. Hum. Mol. Genet. 2001; 10:2861-2867. [PubMed: 11741829]

[7]. Chu K, Koller DL, Snyder R, Fishburn T, Lai D, Waguespack SG, Foroud T, Econs MJ. Analysis of variation in expression of autosomal dominant osteopetrosis type 2: searching for modifier genes. Bone. 2005; 37:655-661. [PubMed: 16120485]

[8]. Brandt S, Jentsch TJ. ClC-6 and ClC-7 are two novel broadly expressed members of the CLC chloride channel family. FEBS Lett. 1995; 377:15-20. [PubMed: 8543009]

[9]. Leisle L, Ludwig CF, Wagner FA, Jentsch T,J, Stauber T. ClC-7 is a slowly voltage-gated 2Cl(-)/ $1 \mathrm{H}(+)$-exchanger and requires Ostm1 for transport activity. EMBO J. 2011; 30:2140-2152. [PubMed: 21527911]

[10]. Stauber T, Weinert S, Jentsch TJ. Cell biology and physiology of CLC chloride channels and transporters. Compr. Physiol. 2012; 2:1701-1744. [PubMed: 23723021]

[11]. Weinert S, Jabs S, Supanchart C, Schweizer M, Gimber N, Richter M, Rademann J, Stauber T, Kornak U, Jentsch TJ. Lysosomal pathology and osteopetrosis upon loss of H+-driven lysosomal Cl- accumulation. Science. 2010; 328:1401-1403. [PubMed: 20430974]

[12]. Kornak U, Kasper D, Bösl MR, Kaiser E, Schweizer M, Schulz A, Friedrich W, Delling G, Jentsch TJ. Loss of the ClC-7 chloride channel leads to osteopetrosis in mice and man. Cell. 2001; 104:205-215. [PubMed: 11207362]

[13]. Schulz P, Werner J, Stauber T, Henriksen K, Fendler K. The G215R mutation in the Cl-/H+antiporter ClC-7 found in ADO II osteopetrosis does not abolish function but causes a severe trafficking defect. PLoS One. 2010; 5:e12585. [PubMed: 20830208]

[14]. Henriksen K, Gram J, Schaller S, Dahl BH, Dziegiel MH, Bollerslev J, Karsdal MA. Characterization of osteoclasts from patients harboring a G215R mutation in ClC-7 causing autosomal dominant osteopetrosis type II. Am. J. Pathol. 2004; 164:1537-1545. [PubMed: 15111300]

[15]. Bouxsein ML, Boyd SK, Christiansen BA, Guldberg RE, Jepsen KJ, Müller R. Guidelines for assessment of bone microstructure in rodents using micro-computed tomography. J. Bone Miner. Res. 2010; 25:1468-1486. [PubMed: 20533309]

[16]. Dempster DW, Compston JE, Drezner MK, Glorieux FH, Kanis JA, Malluche H, Meunier PJ, Ott SM, Recker RR, Parfitt AM. Standardized nomenclature, symbols, and units for bone 
histomorphometry: a 2012 update of the report of the ASBMR Histomorphometry Nomenclature Committee. J. Bone Miner. Res. 2013; 28:1-16. [PubMed: 23255454]

[17]. Rucci N, Rufo A, Alamanou M, Capulli M, Del Fattore A, Ahrman E, Capece D, Iansante V, Zazzeroni F, Alesse E, Heinegård D, Teti A. The glycosaminoglycan-binding domain of PRELP acts as a cell type-specific NF-kappaB inhibitor that impairs osteoclastogenesis. J. Cell Biol. 2009; 187:669-683. [PubMed: 19951916]

[18]. Chu K, Snyder R, Econs MJ. Disease status in autosomal dominant osteopetrosis type 2 is determined by osteoclastic properties. J. Bone Miner. Res. 2006; 21:1089-1097. [PubMed: 16813529]

[19]. Del Fattore A, Cappariello A, Teti A. Genetics, pathogenesis and complications of osteopetrosis. Bone. 2008; 42:19-29. [PubMed: 17936098]

[20]. Frattini A, Pangrazio A, Susani L, Sobacchi C, Mirolo M, Abinun M, Andolina M, Flanagan A, Horwitz EM, Mihci E, Notarangelo LD, Ramenghi U, Teti A, Van Hove J, Vujic D, Young T, Albertini A, Orchard PJ, Vezzoni P, Villa A. Chloride channel ClCN7 mutations are responsible for severe recessive, dominant, and intermediate osteopetrosis. J. Bone Miner. Res. 2003; 18:1740-1747. [PubMed: 14584882]

[21]. Kasper D, Planells-Cases R, Fuhrmann JC, Scheel O, Zeitz O, Ruether K, Schmitt A, Poët M, Steinfeld R, Schweizer M, Kornak U, Jentsch TJ. Loss of the chloride channel ClC-7 leads to lysosomal storage disease and neurodegeneration. EMBO J. 2005; 24:1079-1091. [PubMed: 15706348]

[22]. Chalhoub N, Benachenhou N, RajapurohitaV V, Pata M, Ferron M, Frattini A, Villa A, Vacher J. Grey-lethal mutation induces severe malignant autosomal recessive osteopetrosis in mouse and human. Nat Med. 2003; 9:399-406. [PubMed: 12627228]

[23]. Pangrazio A, Pusch M, Caldana E, Frattini A, Lanino E, Tamhankar PM, Phadke S, Lopez AG, Orchard P, Mihci E, Abinun M, Wright M, Vettenranta K, Bariae I, Melis D, Tezcan I, Baumann C, Locatelli F, Zecca M, Horwitz E, Mansour LS, Van Roij M, Vezzoni P, Villa A, Sobacchi C. Molecular and clinical heterogeneity in CLCN7-dependent osteopetrosis: report of 20 novel mutations. Hum. Mutat. 2010; 31:E1071-1080. [PubMed: 19953639]

[24]. Weinert S, Jabs S, Supanchart C, Schweizer M, Gimber N, Richter M, Rademann J, Stauber T, Kornak U, Jentsch TJ. Lysosomal pathology and osteopetrosis upon loss of H+-driven lysosomal Cl- accumulation. Science. 2010; 328:1401-1403. [PubMed: 20430974]

[26]. Bollerslev J, Nielsen HK, Larsen HF, Mosekilde L. Biochemical evidence of disturbed bone metabolism and calcium homeostasis in two types of autosomal dominant osteopetrosis. Acta Med. Scand. 1988; 224:479-483. [PubMed: 3264447]

[27]. Bénichou OD, Laredo JD, de Vernejoul MC. Type II autosomal dominant osteopetrosis (AlbersSchönberg disease): clinical and radiological manifestations in 42 patients. Bone. 2000; 26:8793. [PubMed: 10617161]

[28]. Dutzler R, Campbell EB, Cadene M, Chait BT, MacKinnon R. X-ray structure of a ClC chloride channel at 3.0 A reveals the molecular basis of anion selectivity. Nature. 2002; 415:287-294. [PubMed: 11796999]

[29]. Leisle L, Ludwig CF, Wagner FA, Jentsch TJ, Stauber T. ClC-7 is a slowly voltage-gated 2Cl(-)/ $1 \mathrm{H}(+)$-exchanger and requires Ostm1 for transport activity. EMBO J. 2011; 30:2140-2152. [PubMed: 21527911] 


\section{Highlights}

- University of L'Aquila (Italy) and Indiana University (USA) have generated the very first animal models of ADO2

- We inserted in C57BL6/J6 mouse the homolog (p.G213R) of the most frequent mutation of the human CLCN7 gene (p.G215R)

- This mutation is pathogenic in homozygous and heterozygous mice, this latter showing autosomal dominant inheritance and ADO2 phenotype

- We cross-bred our ADO2 C57BL6/J6 mice with mice of various other backgrounds

- We observed phenotypic variability consistent with incomplete penetrance and presence of modifier genes that could affect $\mathrm{ADO} 2$ severity 

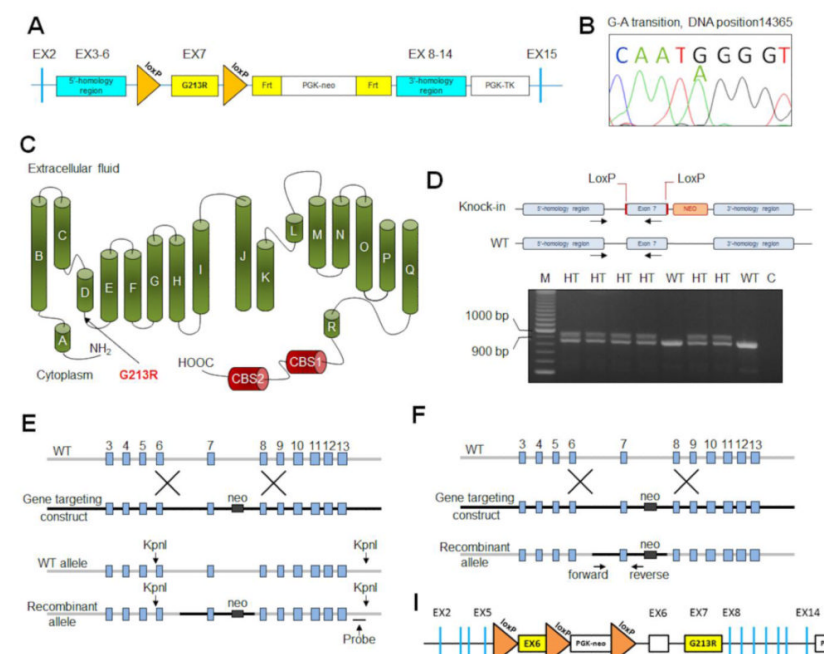

$\mathbf{F}$
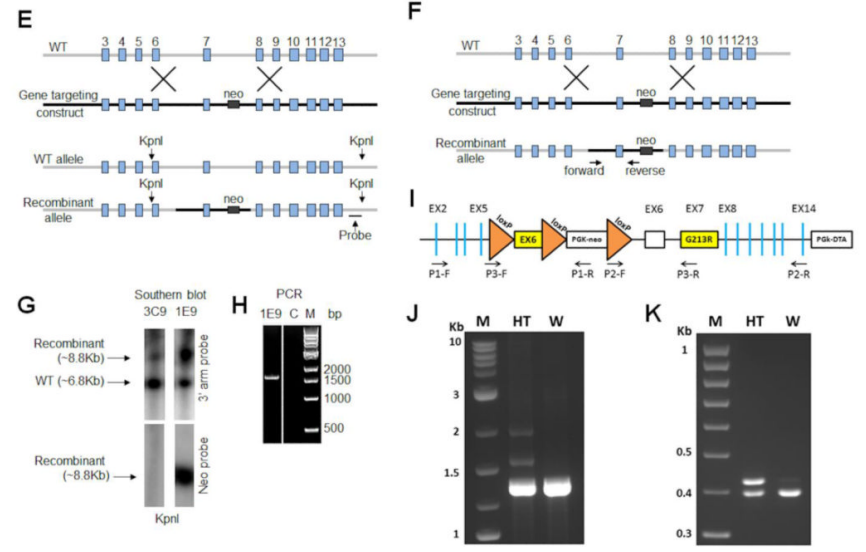

Figure 1. Generation of p.G213R-Clcn7 knock-in B6 mice

University of L'Aquila: (A) Gene targeting pFlrt1 vector for the p.G213R-Clcn7 knock-in mouse. $5^{\prime}$ and $3^{\prime}$ homology regions were cloned upstream and downstream of the exon 7 , respectively, to promote the homologous recombination. LoxP: locus of X-over P1; Frt: flippase recognition target site; PGK: phopshoglycerate kinase promoter; neo: neomycin; TK: thymidine kinase. (B) Electropherogram to confirm insertion of G-A transition at position 14365 of the DNA. (C) Cartoon illustrating CLC-7 protein subunit conformation and position of p.G213R mutation [28, 29]. CBS: cystathionine $\beta$-synthase domain. (D) PCR strategy (upper panel) for F1 mouse genotyping (lower panel). Arrows indicate primers sites for PCR. WT: wild-type; HT: heterozygous. M: molecular weight markers; bp: base pair; C: negative control. (E) Southern blot strategy for $3^{\prime}$ recombination based on DNA digestion by KpnI restriction enzyme, to discriminate between the wild-type (WT) allele ( $\sim 6.8 \mathrm{~kb})$ and the recombinant allele, which was $2 \mathrm{~kb}(\sim 8.8 \mathrm{~kb})$ longer because of the presence of the Neo cassette. (F) PCR strategy for $5^{\prime}$ screening using primers annealing to pFlrt1 vector sequences just upstream and downstream the region containing exon 7, to obtain the expected recombinant band of $\sim 1.7 \mathrm{~kb}$. (G) Southern blot for $3^{\prime}$ recombination of ES clone 1E9. 3C9 is a non recombinant clone used as negative control. Kb: kilobase. (H) PCR for $5^{\prime}$ recombination of ES clone 1E9. C: negative control; M: molecular weight markers; bp: base pair. Indiana University: (I) Gene targeting by homologous recombination technique to insert the p.G213R mutation in exon 7 and Cre-loxP recombination strategy to remove neomycin from the targeted allele: construct containing exon 6 with a stop codon (EX6 highlighted) flanked by loxP sites, the neomycin resistance gene flanked by loxP sites, as well as the p.G213R mutation (highlighted) in exon 7 inserted into ES cells (CCE916) from $129 \mathrm{~Sv}$ mouse. Screening of positive clones using primers at $5^{\prime}$-end (P1-F and P1-R) and at $3^{\prime}$-end (P2-F and P2-R) produced bands at 3626 and 5969 bp (data not shown), respectively. (J) Excision of the additional exon 6 and neomycin cassette using primers (P3-F and P3-R) produced band only at $\sim 1.3 \mathrm{~kb}$ for wild-type mice and $\sim 1.3, \sim 1.6$ and $\sim 1.9 \mathrm{~kb}$ bands for 
heterozygote mutant mice. (K) Restriction fragment length polymorphism detection of mutant exon 7: wild-type mice with $389 \mathrm{bp}$ band and mutant mice with $389 \mathrm{bp}$ and $419 \mathrm{bp}$ bands. M: molecular marker; WT: wild-type; HT: heterozygous; Kb: kilobase; PGK: phopsho-glycerate kinase promoter; neo: neomycin; DTA: diphtheria toxin A fragment gene. 
A

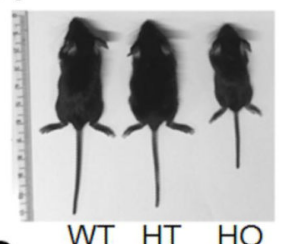

D

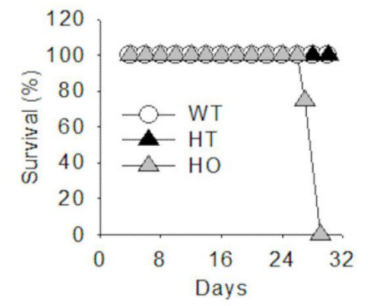

F

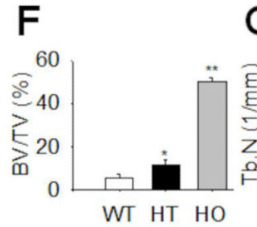

G
B $\quad$ C

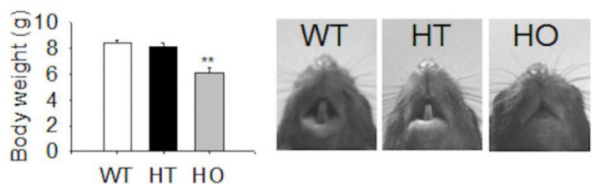

E
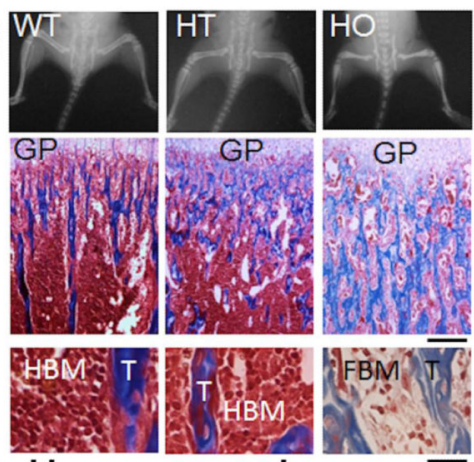

$\mathrm{H}$

Figure 2. B6 mouse phenotyping

Thirty days old wild-type (WT), heterozygous (HT) and homozygous (HO) B6 mice were sacrificed and evaluated for (A) gross appearance, (B) body weight (B6) $(n=3 ; * * p<0.02)$, (C) tooth eruption and (D) survival (B6). (E) X-ray analysis of hindlimbs, pelvis and vertebrae (upper panels) and histological examination of tibia proximal spongiosa (Masson's trichrome staining) (middle and lower panels). GP: growth plate. HBM: hematopoietic bone marrow. FBM: fibrotic bone marrow. T: trabecula. Middle panels; bar $=200 \mu \mathrm{m}$. Lower panels: bar $=30 \mu \mathrm{m}$. Histomorphometric evaluation of proximal tibia $(\mathrm{F})$ trabecular bone volume/total tissue volume (BV/TV), $(\mathrm{G})$ trabecular number $(\mathrm{Tb} . \mathrm{N}),(\mathrm{H})$ trabecular thickness (Tb.Th) and (I) trabecular separation (Tb.Sp). Data are the mean \pm s.d. of 3 mice per group; $* \mathrm{p}<0.05 ; * * \mathrm{p}<0.002$. 

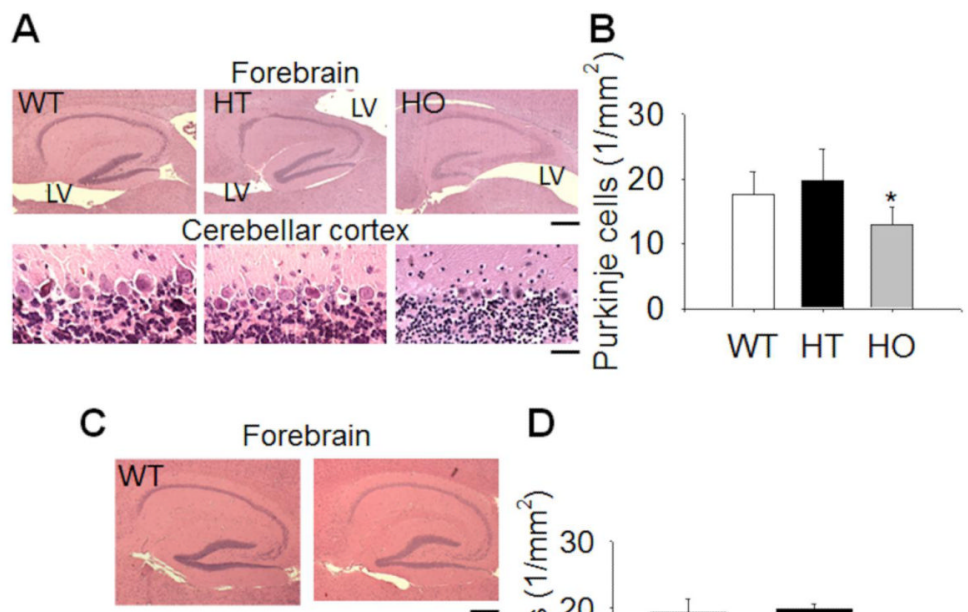

Forebrain
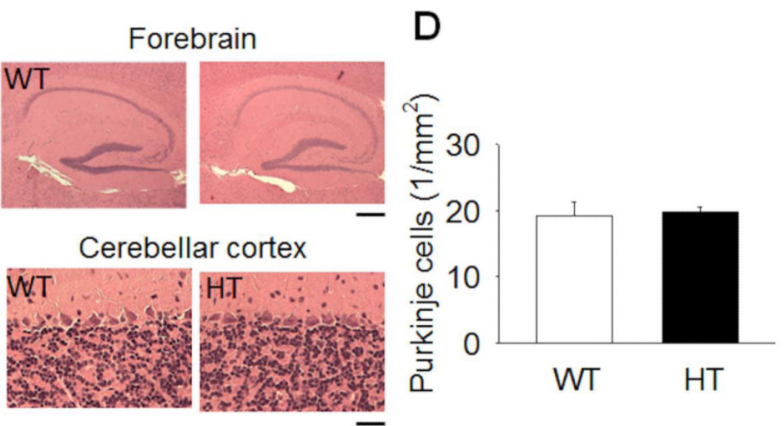

Figure 3. Neuronal phenotype in B6 mice

(A) Histological examination (hematoxylin/eosin staining) showing neuronal loss in the hippocampal regions (upper panels, Bar $=400 \mu \mathrm{m}$ ) and in the cerebellar cortex (lower panels, Bar $=60 \mu \mathrm{m}$ ) of 1 month old mice. LV: lateral ventricle. (B) Quantification of cerebellar Purkinje cells. Data are the mean \pm s.d. of 3 mice per group; ${ }^{*} \mathrm{p}<0.02$. (C)

Hematoxylin/eosin staining of the hippocampal regions (upper panels, Bar $=400 \mu \mathrm{m}$ ) and cerebellar cortex (lower panels, Bar $=60 \mu \mathrm{m}$ ) of 12 months old mice. WT: wild-type. HT: heterozygous. (D) Quantification of cerebellar Purkinje cells. Data are the mean \pm s.d. of 3 mice per group. Differences are statistically not significant $(\mathrm{p}=0.72)$. 

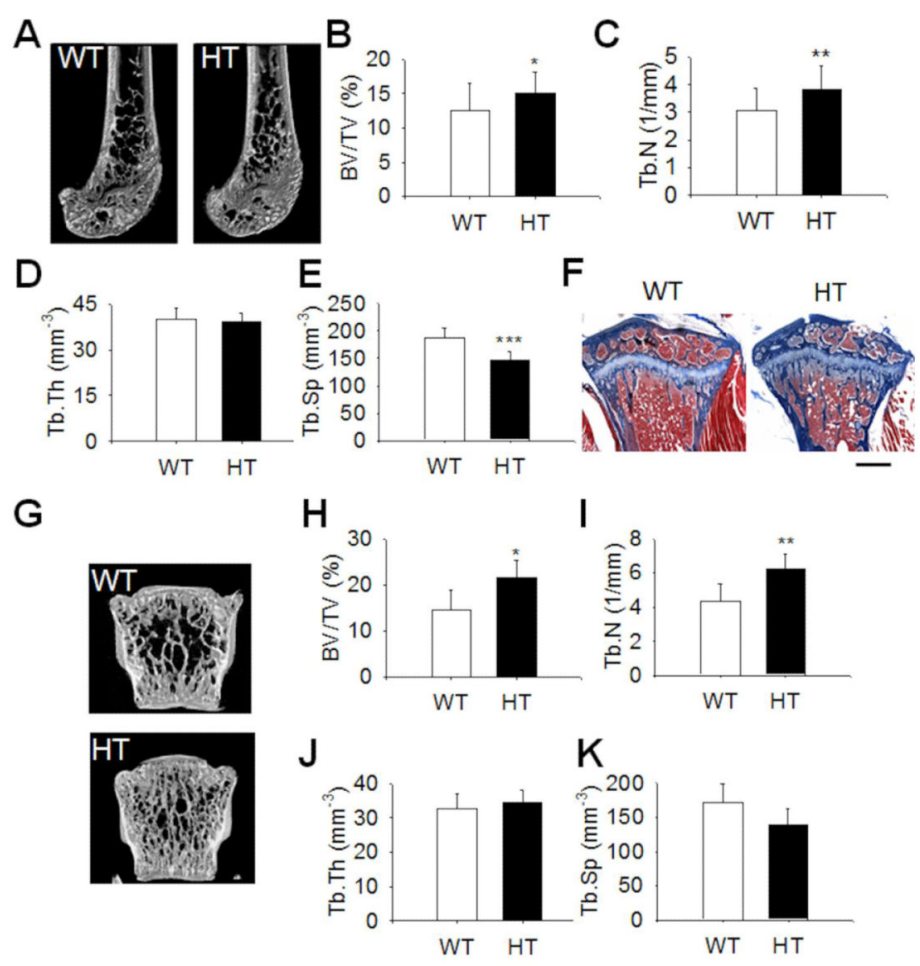

Figure 4. Bone structural parameters of 3 months old wild-type (WT) and heterozygous (HT) p.G213R-Clcn7 knock-in B6 ADO2 mice

(A) $\mu \mathrm{CT}$ images of distal femurs. (B) Trabecular bone volume (BV/TV), (C) number (Tb.N), (D) thickness (Tb.Th) and (E) separation (Tb.Sp). (F) Masson's trichromic staining of proximal tibia. Bar $=500 \mu \mathrm{m}$. $(\mathrm{G}) \mu \mathrm{CT}$ images of $\mathrm{L} 2$ vertebra. $(\mathrm{H})$ Vertebral trabecular bone volume (BV/TV), (I) number (Tb.N), (J) thickness (Tb.Th) and (K) separation (Tb.Sp). Data are representative $(A, F, G)$ or the mean \pm s.d. (B-E, H-K) of 4-16 mice/group. In (B-E) $* \mathrm{p}=0.046 ; * * \mathrm{p}=0.014 ; * * \mathrm{*}=0.0000005$. In (H-I) $* \mathrm{p}=0.021 ; * \mathrm{p}=0.014$. In (D,J,K) data are statistically not significant $(\mathrm{p}>0.3)$. 
A

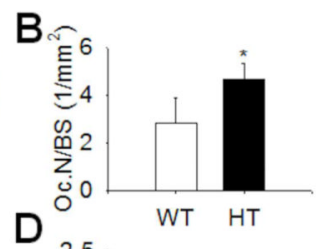

C

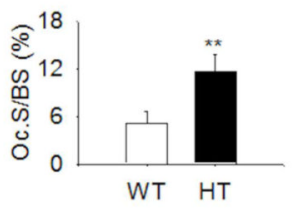

E

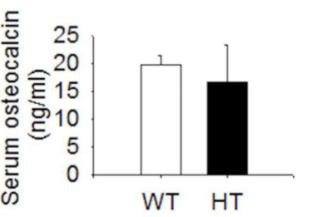

G

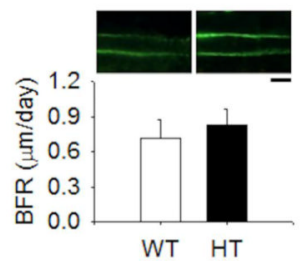

|

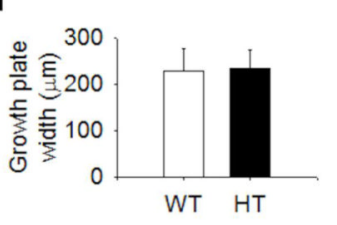

E
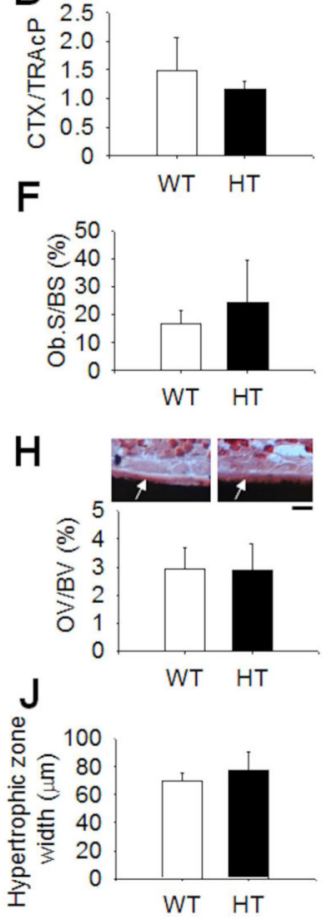

Figure 5. Bone cellular and dynamic parameters of 3 months old wild-type (WT) and heterozygous (HT) p.G213R-Clcn7 knock-in B6 ADO2 mice

(A) TRAcP histochemical staining (arrows) of calvariae. (B) Osteoclast number (Oc.N) and (C) osteoclast surface (Oc.S)/bone surface (BS) in proximal tibiae. (D) Serum CTX/TRAcP ratio. (E) ELISA assay of serum levels of the osteoblast-specific protein, osteocalcin.

Histomorphometric evaluation of $(\mathrm{F})$ osteoblast surface/bone surface $(\mathrm{Ob} . \mathrm{S} / \mathrm{BS}),(\mathrm{G})$ bone formation rate (BFR) (upper panels: green fluorescence of calcein labels; Bar=20 $\mu \mathrm{m}),(\mathrm{H})$ osteoid volume over bone volume (OV/BV) (upper panels: von Kossa/acidic fuchsin staining; arrow: osteoid; Bar=20 $\mathrm{m}$ ), (I) total growth plate width and (J) growth plate hypertrophic zone width. Data are representative (A) or the mean \pm s.d. (B-J) of 4 mice/ group. ${ }^{*} \mathrm{p}<0.005 ; *{ }^{*}<<0.006$. In $(\mathrm{E}-\mathrm{H})$ differences are not statistically significant $(\mathrm{p}=>0.35)$. 

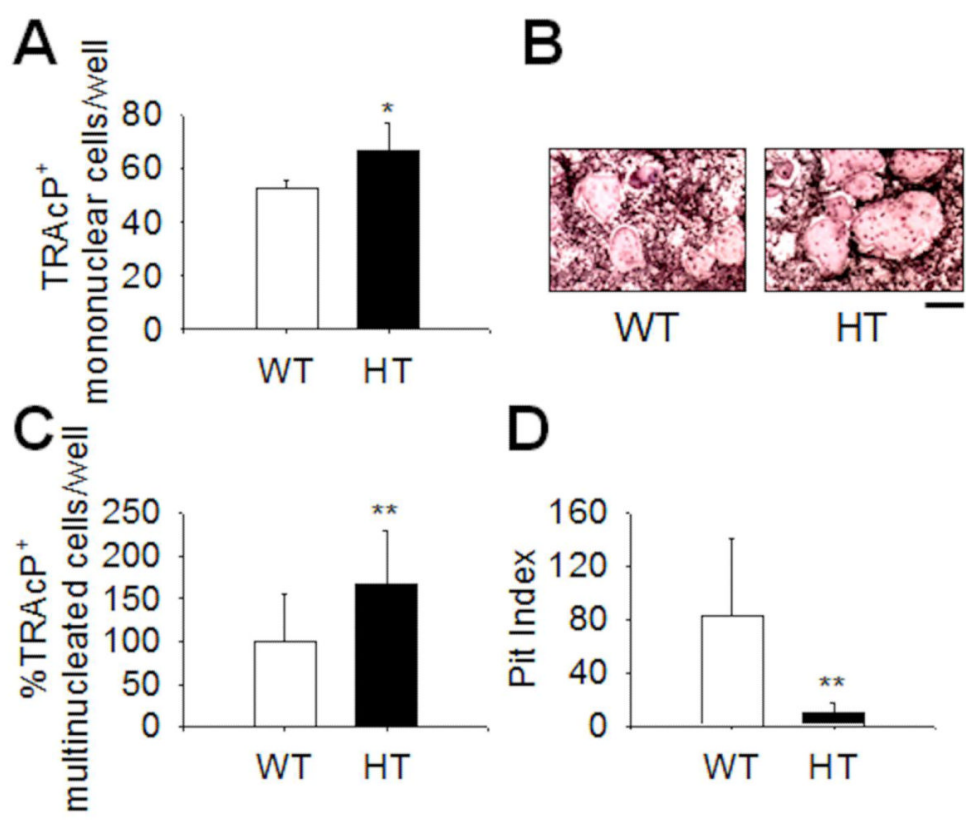

Figure 6. Ex vivo osteoclasts

(A) Quantification of bone marrow $\mathrm{TRAcP}^{+}$mononuclear cells in bone marrow freshly flushed off from the hindlimb bone cavities. (B) Bone marrow osteoclast precursors were cultured for 6 days in the presence of M-CSF and RANKL and stained cytochemically to (B) detect and (C) enumerate the $\mathrm{TRAcP}^{+}$multinucleated osteoclasts (range of osteoclast number in WT=46-78/well). Bar=30 $\mu \mathrm{m}$. (D) Quantification of bone resorption (pit assay) by osteoclasts differentiated onto bovine bone slices. Data are (B) representative or (A,C,D) the mean \pm s.d. of 3 independent experiments. *p<0.04; **p $<0.002$. 


\section{Table 1}

$\mu \mathrm{CT}$ analysis of femoral and vertebral trabecular bone in aging wild-type (WT) and heterozygous (HT) B6 ADO2 mice.

\begin{tabular}{|c|c|c|c|c|c|}
\hline Femur & $\begin{array}{c}\text { Age } \\
\text { (months) }\end{array}$ & $\mu \mathrm{CT}$ parameters & $\begin{array}{l}\text { WT } \\
n=4\end{array}$ & $\begin{array}{l}\text { HT } \\
n=3\end{array}$ & p-value \\
\hline & \multirow{4}{*}{6} & BV/TV (\%) & $10.25 \pm 3.47$ & $24.27 \pm 0.40$ & $0.03^{*}$ \\
\hline & & Tb.N (1/mm) & $2.55 \pm 0.07$ & $5.52 \pm 1.83$ & $0.03 *$ \\
\hline & & Tb.Th (mm) & $0.040 \pm 0.0018$ & $0.043 \pm 0.0029$ & 0.10 \\
\hline & & $\mathrm{Tb} . \mathrm{Sp}(\mathrm{mm})$ & $0.214 \pm 0.067$ & $0.127 \pm 0.038$ & 0.10 \\
\hline & \multirow{4}{*}{12} & BV/TV (\%) & $5.57 \pm 2.52$ & $9.86 \pm 2.80$ & $0.01^{*}$ \\
\hline & & Tb.N (1/mm) & $1.42 \pm 0.69$ & $2.37 \pm 0.58$ & $0.04^{*}$ \\
\hline & & Tb.Th (mm) & $0.040 \pm 0.0024$ & $0.041 \pm 0.0032$ & 0.43 \\
\hline & & Tb.Sp (mm) & $0.255 \pm 0.073$ & $0.183 \pm 0.019$ & $0.02^{*}$ \\
\hline \multirow[t]{9}{*}{ L2 Vertebra } & $\begin{array}{c}\text { Age } \\
\text { (months) }\end{array}$ & $\mu \mathrm{CT}$ parameters & $\begin{array}{l}\text { WT } \\
n=4\end{array}$ & $\begin{array}{l}\text { HT } \\
n=3\end{array}$ & p-value \\
\hline & \multirow{4}{*}{6} & BV/TV (\%) & $10.49 \pm 1.68$ & $14.53 \pm 1.11$ & $0.02^{*}$ \\
\hline & & Tb.N (1/mm) & $3.68 \pm 0.34$ & $4.82 \pm 0.25$ & $0.01^{*}$ \\
\hline & & Tb.Th (mm) & $0.028 \pm 0.0022$ & $0.030 \pm 0.004$ & 0.51 \\
\hline & & Tb.Sp (mm) & $0.176 \pm 0.0066$ & $0.154 \pm 0.0013$ & 0.057 \\
\hline & \multirow{4}{*}{12} & $\mathrm{BV} / \mathrm{TV}(\%)$ & $7.36 \pm 1.43$ & $11.33 \pm 3.14$ & $0.026^{*}$ \\
\hline & & Tb.N (1/mm) & $3.27 \pm 0.70$ & $4.55 \pm 1.21$ & 0.067 \\
\hline & & Tb.Th (mm) & $0.023 \pm 0.002$ & $0.025 \pm 0.0032$ & 0.15 \\
\hline & & Tb.Sp (mm) & $0.199 \pm 0.054$ & $0.164 \pm 0.035$ & 0.19 \\
\hline
\end{tabular}




\section{Table 2}

$\mu \mathrm{CT}$ analysis of femoral trabecular bone of 3 months old male and female wild-type (WT) and heterozygous (HT) B6 ADO2 mice.

\begin{tabular}{lcccc}
\hline Gender & $\mu$ CT parameters & $\begin{array}{c}\text { WT } \\
\mathbf{n = 8}\end{array}$ & $\begin{array}{c}\text { HT } \\
\mathbf{n = 7 - 8}\end{array}$ & p-value \\
\hline \multirow{2}{*}{ Male } & BV/TV (\%) & $15.87 \pm 1.45$ & $17.02 \pm 0.93$ & $0.047^{*}$ \\
& Tb.N (1/mm) & $3.69 \pm 0.45$ & $4.37 \pm 0.70$ & $0.035^{*}$ \\
& Tb.Th (mm) & $0.043 \pm 0.0019$ & $0.039 \pm 0.0020$ & $0.001^{*}$ \\
& Tb.Sp (mm) & $0.178 \pm 0.013$ & $0.135 \pm 0.012$ & $0.000008^{*}$ \\
\hline \multirow{2}{*}{ Female } & BV.N $(1 / \mathrm{Tm})$ & $2.30 \pm 0.35$ & $3.28 \pm 0.61$ & $0.015^{*}$ \\
& Tb.Th (mm) & $0.0374 \pm 0.003$ & $0.0397 \pm 0.0035$ & 0.138 \\
& Tb.Sp (mm) & $0.198 \pm 0.0183$ & $0.157 \pm 0.0154$ & $0.0004^{*}$ \\
\hline
\end{tabular}

* indicates statistically significant differences 


\section{Table 3}

Whole body areal Bone Mineral Density (aBMD) in the indicated mouse strains crossbred with B6 ADO2 mice. HT: heterozygous mice; WT: wild-type mice

\begin{tabular}{lcccc}
\hline $\begin{array}{l}\text { Mouse strains } \\
\text { (generation) }\end{array}$ & $\begin{array}{c}\text { WT } \\
\mathbf{n}=13-17\end{array}$ & $\begin{array}{c}\text { HT } \\
\mathbf{n}=12-14\end{array}$ & $\begin{array}{c}\text { aBMD } \\
\text { \% increase }\end{array}$ & p-value \\
\hline 129 (N5) & $0.052 \pm 0.002$ & $0.056 \pm 0.002$ & 8 & $0.0003^{*}$ \\
D2 (N5) & $0.048 \pm 0.002$ & $0.050 \pm 0.002$ & 4 & $0.02^{*}$ \\
Balb/c (F1) & $0.052 \pm 0.003$ & $0.054 \pm 0.003$ & 3.8 & 0.1 \\
\hline * & & &
\end{tabular}




\section{Table 4}

$\mu \mathrm{CT}$ analysis of distal femur trabecular bone volume and structural parameters in 3 months old wild-type (WT) and heterozygous (HT) mice of the indicated strains cross-bred with B6 ADO2 mice.

\begin{tabular}{|c|c|c|c|c|}
\hline $\begin{array}{l}\text { Mouse strains } \\
\text { (generation) }\end{array}$ & $\mu \mathrm{CT}$ parameters & $\begin{array}{c}\text { WT } \\
n=5-16\end{array}$ & $\underset{n=5-13}{\text { HT }}$ & p-value \\
\hline \multirow{4}{*}{129 (N5) } & $\mathrm{BV} / \mathrm{TV}(\%)$ & $7.200 \pm 0.800$ & $14.10 \pm 1.40$ & $0.0002^{*}$ \\
\hline & Tb.N (1/mm) & $2.317 \pm 0.180$ & $4.009 \pm 0.244$ & $<0.0001^{*}$ \\
\hline & Tb.Th (mm) & $0.030 \pm 0.001$ & $0.034 \pm 0.001$ & $0.03^{*}$ \\
\hline & Tb.Sp (mm) & $0.445 \pm 0.050$ & $0.225 \pm 0.016$ & $0.0005^{*}$ \\
\hline \multirow{4}{*}{ D2 (N5) } & $\mathrm{BV} / \mathrm{TV}(\%)$ & $6.100 \pm 0.600$ & $8.800 \pm 0.700$ & $0.008^{*}$ \\
\hline & Tb.N (1/mm) & $1.172 \pm 0.154$ & $2.584 \pm 0.194$ & $0.002^{*}$ \\
\hline & Tb.Th (mm) & $0.035 \pm 0.001$ & $0.034 \pm 0.001$ & 0.44 \\
\hline & Tb.Sp (mm) & $0.626 \pm 0.070$ & $0.381 \pm 0.034$ & $0.007^{*}$ \\
\hline \multirow{4}{*}{ Balb/c (F1) } & $\mathrm{BV} / \mathrm{TV}(\%)$ & $13.600 \pm 1.900$ & $16.400 \pm 2.000$ & $0.03^{*}$ \\
\hline & Tb.N (1/mm) & $3.168 \pm 0.345$ & $4.263 \pm 0.449$ & 0.06 \\
\hline & Tb.Th (mm) & $0.041 \pm 0.002$ & $0.038 \pm 0.001$ & 0.26 \\
\hline & Tb.Sp (mm) & $0.339 \pm 0.042$ & $0.236 \pm 0.038$ & 0.09 \\
\hline \multirow{4}{*}{ CD1 (F1) } & BV/TV (\%) & $5.280 \pm 1.560$ & $8.420 \pm 3.610$ & 0.11 \\
\hline & Tb.N (1/mm) & $1.180 \pm 0.290$ & $2.210 \pm 0.980$ & 0.12 \\
\hline & Tb.Th (mm) & $0.045 \pm 0.002$ & $0.038 \pm 0.002$ & 0.05 \\
\hline & Tb.Sp (mm) & $0.191 \pm 0.021$ & $0.154 \pm 0.035$ & 0.12 \\
\hline
\end{tabular}




\section{Table 5}

Serum bone resorption biomarker collagen type 1 cross-linked C Telopeptide (CTX) normalized to serum Tartrate-Resistant Acid Phospahatase (TRAcP) (CTX/TRAcP), in heterozygous (HT) and wild-type (WT) mice of the indicated strains cross-bred with B6 ADO2 mice.

\begin{tabular}{lccc}
\hline $\begin{array}{l}\text { Mouse strains } \\
\text { (generation) }\end{array}$ & \multicolumn{3}{c}{ CTX/TRAcP } \\
\cline { 2 - 4 } & $\begin{array}{c}\text { WT } \\
\mathbf{N}=(\mathbf{6 - 1 7})\end{array}$ & $\begin{array}{c}\text { HT } \\
\mathbf{N}=(\mathbf{6 - 1 4})\end{array}$ & p-value \\
\hline 129 (N5) & $1.646 \pm 0.167$ & $0.776 \pm 0.150$ & $0.0008^{*}$ \\
D2 (N5) & $1.661 \pm 0.147$ & $1.398 \pm 0.105$ & 0.17 \\
Balb/c (F1) & $3.635 \pm 0.436$ & $2.126 \pm 0.183$ & $0.005^{*}$ \\
CD1 (F1) & $3.489 \pm 0.205$ & $2.173 \pm 0.160$ & $0.004^{*}$ \\
\hline
\end{tabular}

*indicates statistically significant differences 